\section{Fatores de risco para transmissão do HIV em usuários de drogas de Porto Alegre, Rio Grande do Sul, Brasil}

\author{
Risk factors for HIV transmission in drug users \\ from Porto Alegre, Rio Grande do Sul State, Brazil
}

\author{
1 Faculdade de Medicina, \\ Universidade Federal \\ do Rio Grande do Sul, \\ Porto Alegre, Brasil. \\ 2 Center for Drug and \\ Alcohol Studies, University \\ of Delaware, Newark, U.S.A. \\ Correspondência \\ L. von Diemen \\ Centro de Pesquisas \\ em Álcool e Drogas, \\ Departamento \\ de Psiquiatria, Faculdade \\ de Medicina, Universidade \\ Federal do Rio Grande \\ do Sul. Rua Ramiro \\ Barcelos 2400, 4o andar, \\ Porto Alegre, RS \\ 90035-003, Brasil. \\ lisiavd@hotmail.com
}

\begin{abstract}
A cross-sectional study with a sample of 420 drug users from Porto Alegre, Rio Grande do Sul State, Brazil, was utilized to assess demographic variables, drug use, and risk behaviors for HIV infection. We used the Brazilian version of the Risk Assessment Battery. Overall HIV seropositivity was 22.6\%; 39.3\% of the subjects infected were at least 30 years old, and $69.5 \%$ were males. In the month prior to the interview, 56.8\% of the sample had used marijuana, 43.6\% had sniffed cocaine, $17.6 \%$ had injected cocaine, and $42.4 \%$ had used alcohol on a frequent basis. The variables that continued to be associated with HIV infection after logistic regression were age (30 or older) (OR: 2.89; 95\%CI: 1.17-7.12), having less than seven years of schooling (OR: 2.10; 95\%CI: 1.02-4.36), having a monthly family income of less than one minimum wage, or approximately U\$90 (OR: 2.89; 95\%CI: 1.32-6.32), and having injected drugs (OR: 5.18; 95\%CI: 1.32-6.32). Seroprevalence in this sample is considered high, particularly since $70.0 \%$ of the sample reported no prior drug injection. Variables associated with HIV infection are similar to the national and international literature and agree with the theoretical model of risk behavior proposed by the first author.
\end{abstract}

HIV; Acquired Immunodeficiency Syndrome; Street Drugs; Risk Factors
Flavio Pechansky 1 Lisia Von Diemen 1 James A. Inciardi 2 Hilary Surratt 2 Raquel De Boni 1

\section{Introdução}

A transmissão do HIV entre os usuários de drogas se dá pela via sangüínea, por meio de compartilhamento de agulhas, seringas e demais equipamentos para uso de drogas endovenosas, e pela via sexual. Dessa forma, os usuários de drogas injetáveis (UDI) estão expostos à dupla via de contaminação e se constituem em importantes transmissores do HIV para seus parceiros sexuais, usuários de drogas injetáveis ou não, como apontam Friedman et al. 1 .

Paralelamente a isso, os usuários de drogas se expõem mais a situações de risco sexual, como mostram Anderson et al. ${ }^{2}$. Edlin et al. ${ }^{3}$ estudaram a transmissão do HIV entre usuários de crack que nunca haviam injetado drogas, cuja prevalência de infecção foi de 15,7\%, comparada com indivíduos que nunca usaram crack, com soropositividade de 5,2\%. Após a análise multivariada, as variáveis associadas com infecção foram as de risco sexual, como troca de sexo por droga ou dinheiro, relação sexual anal e não-uso de preservativo. Inciardi et al. 4 demonstram a associação que há entre o uso de crack e a maior contaminação por HIV e descrevem como as mulheres usuárias desta droga se prostituem a fim de obtê-la, tornando-se sujeitos de altíssimo risco para a transmissão do vírus.

No nosso meio existem poucos estudos que avaliam as situações de risco para a transmissão do HIV entre os usuários de drogas, apesar 
dos dados do Boletim Epidemiológico - AIDS (abril a setembro de 2002 - http: / / www.aids.gov. br, acessado em 07/Jul/2003) no Brasil apontarem para o uso de drogas injetáveis como a via de contaminação de $15,8 \%$ dos homens e de $6,1 \%$ das mulheres maiores de 13 anos no ano de 2002. A prevalência de infecção por HIV em UDI encontrada por Pechansky et al. 5,6 em dois estudos realizados em Porto Alegre foi de $54,5 \%$ e de $44,5 \%$. No primeiro estudo, quando os UDI foram excluídos da análise, a infecção nos usuários de drogas em geral foi de $16,0 \% 7$. No Rio de Janeiro, Lima et al. 8, em um estudo com amostra de UDI recrutados tanto nas ruas quanto em centros de saúde encontraram infecção em $34,0 \%$ dos casos. É importante salientar também o elevado índice de uso de drogas na Região Sul do Brasil, que em relatório divulgado pela Secretaria Nacional Anti-drogas (1o Levantamento Nacional sobre o Uso de Drogas - http://www.senad.gov.br, acessado em 25/Jul/2002) desponta como a maior prevalência de uso na vida de qualquer droga exceto álcool e tabaco $(17,1 \%)$, dependência de maconha $(1,6 \%)$, uso na vida de maconha $(8,4 \%)$ e cocaína $(3,6 \%)$ em todo o país. Além disso, Porto Alegre é hoje a terceira cidade brasileira com maior número de casos acumulados de AIDS, totalizando $4,1 \%$ dos casos totais do país, e a cidade com maior taxa de incidência de casos novos de AIDS, com 49,1/100 mil habitantes no ano de 2002, de acordo com o Boletim Epidemiológico de AIDS (abril a setembro de $2002-$ http://www.aids.gov.br, acessado em 07/Jul/ 2003).

O objetivo desse artigo é descrever as características sócio-demográficas e as situações de risco a que estão expostos os usuários de drogas de Porto Alegre e sua relação com a soropositividade, testando a associação de algumas variáveis de um modelo de exposição ao HIV em usuários de drogas proposto por Pechansky 9 .

\section{Método}

\section{Sujeitos e delineamento}

Foi realizado um estudo transversal com uma amostra de conveniência de 695 voluntários de ambos os sexos, com idades variando entre 15 e 60 anos. Os critérios de inclusão destes sujeitos foram os relatos obtidos por entrevista de triagem de uso de qualquer droga (incluindo álcool e tranqüilizantes). O uso de álcool e tranqüilizantes foi incluído quando de forma freqüente ou diária. Os indivíduos entrevistados eram convidados a indicar conhecidos que também se enquadrassem no critério de idade e uso de substâncias, em um método de amostragem do tipo "bola-de-neve".

\section{Coleta}

Para a coleta, foram treinados 15 entrevistadores, todos profissionais com nível de graduação ou estudantes da área médica. Os locais para coleta foram o Centro de Apoio e Orientação Sorológica (COAS Municipal) Paulo César Bonfim, da Prefeitura Municipal de Porto Alegre, onde os clientes procuram testagens HIV gratuita e anônima (97 casos - 14,0\% do total), o Centro de Orientação e Apoio Sorológico Estadual (COAS Estadual) (242 casos - 34,8\%), onde os clientes também procuram testagem anônima e gratuita, o Ambulatório da Cruz Vermelha do Brasil (234 casos - 33,7\%) e o Ambulatório de Álcool e Drogas do Hospital de Clínica de Porto Alegre - HCPA - (122 casos - 17,6\%), perfazendo um total de 695 casos.

Foram excluídos 275 indivíduos cujo resultado do exame anti-HIV não estava disponível. Essa perda ocorreu especialmente devido à dificuldade em coletar o sangue para o exame no momento da coleta de dados em dois dos quatro locais de coleta, já que no HCPA e na Cruz Vermelha os indivíduos eram encaminhados para a realização do exame em outra data. $\mathrm{Na}$ Tabela 1, foram comparados os indivíduos excluídos com os que tinham o resultado antiHIV. A amostra final de 420 indivíduos que será utilizada nestas análises foi significativamente diferente quanto a ter menos homens incluídos, ter mais indivíduos com trabalho de forma sistemática, ter menos indivíduos sem trabalho, ter mais indivíduos com renda entre $1 \mathrm{e}$ 3 salários mínimos, menos indivíduos com renda acima de 4 salários mínimos, ter menos indivíduos com uso de droga injetável desde 1980. Além disso, os indivíduos que não tinham resultado eram, na sua maioria, provenientes do HCPA e da Cruz Vermelha, pelos motivos já descritos. É provável que o local de origem explique as outras diferenças encontradas, visto que o HCPA e a Cruz Vermelha são locais onde os participantes buscavam tratamento para dependência química, diferente dos COAS, onde buscavam apenas testagem anti-HIV.

\section{Instrumento}

O instrumento utilizado para avaliação das situações de risco nos usuários de drogas foi o questionário CRA (Comportamento de Risco para AIDS), desenvolvido e validado na Universi- 
Comparação entre os indivíduos com e sem resultado do teste anti-HIV disponível.

\begin{tabular}{|c|c|c|c|c|c|}
\hline \multirow[t]{2}{*}{ Variável } & \multicolumn{2}{|c|}{$\begin{array}{l}\text { Sem resultado HIV } \\
(n=275)\end{array}$} & \multicolumn{2}{|c|}{$\begin{array}{l}\text { Com resultado HIV } \\
(n=420)\end{array}$} & \multirow[t]{2}{*}{ p } \\
\hline & & $\%$ & $\mathrm{~N}$ & $\%$ & \\
\hline \multicolumn{6}{|l|}{ Idade (em anos) } \\
\hline$<20$ & 49 & 18,4 & 67 & 16,3 & 0,49 \\
\hline $20-29$ & 111 & 41,6 & 185 & 45,0 & 0,38 \\
\hline 30 ou mais & 107 & 40,1 & 159 & 38,7 & 0,72 \\
\hline \multicolumn{6}{|l|}{ Gênero } \\
\hline Homem & 235 & 85,5 & 292 & 69,5 & $<0,001$ \\
\hline Mulher & 40 & 14,5 & 128 & 30,5 & $<0,001$ \\
\hline \multicolumn{6}{|l|}{ Estado marital } \\
\hline Solteiro ou sem companheiro/a regular & 153 & 55,6 & 195 & 46,4 & $<0,05$ \\
\hline Casado ou com companheiro/a regular & 74 & 26,9 & 153 & 36,4 & $<0,05$ \\
\hline Outra situação conjugal & 48 & 17,5 & 70 & 16,7 & 0,81 \\
\hline \multicolumn{6}{|l|}{ Situação profissional } \\
\hline Trabalho sistemático & 113 & 41,1 & 223 & 53,1 & $<0,05$ \\
\hline Trabalho irregular (não sistemático) & 84 & 30,5 & 113 & 27,0 & 0,31 \\
\hline Não trabalha & 78 & 28,4 & 83 & 19,9 & $<0,05$ \\
\hline \multicolumn{6}{|l|}{ Escolaridade (em anos de estudo) } \\
\hline$<7$ & 122 & 44,4 & 171 & 40,8 & 0,33 \\
\hline $8-11$ & 76 & 27,6 & 108 & 25,8 & 0,57 \\
\hline 12 ou mais & 76 & 27,6 & 140 & 33,4 & 0,11 \\
\hline \multicolumn{6}{|l|}{ Renda (em salários mínimos) } \\
\hline$<1$ & 50 & 18,4 & 84 & 20,4 & 0,52 \\
\hline $1-3$ & 83 & 30,5 & 161 & 39,1 & $<0,05$ \\
\hline 4 ou mais & 139 & 51,1 & 167 & 40,5 & $<0,05$ \\
\hline Uso de droga injetável (1980) & 117 & 42,5 & 129 & 30,7 & $<0,05$ \\
\hline \multicolumn{6}{|l|}{ Local de coleta } \\
\hline Hospital de Clínicas de Porto Alegre & 83 & 30,5 & 39 & 9,3 & $<0,05$ \\
\hline Cruz Vermelha & 172 & 63,2 & 60 & 14,3 & $<0,05$ \\
\hline COAS & 17 & 6,2 & 321 & 76,4 & $<0,05$ \\
\hline
\end{tabular}

dade da Pensilvânia por Metzger et al. 10. Uma versão traduzida e adaptada do CRA por Pechansky et al. ${ }^{11}$ para o português foi utilizada. O CRA é um instrumento de fácil entendimento, foi auto-administrado na maioria dos casos e o tempo médio de preenchimento foi de dez minutos. As questões avaliam os dados demográficos do respondente, sua história de uso de drogas, seu estado sorológico para o HIV, seu comportamento sexual, as relações envolvendo sexo, dinheiro e drogas, e suas preocupações referentes à contaminação e transmissão do HIV. O ingresso dos sujeitos neste estudo foi feito mediante consentimento verbal, com a fi- nalidade de preservar a identidade dos participantes. O projeto foi aprovado pelo Conselho Estadual de Entorpecentes e pela Comissão de Ética e Pesquisa do HCPA.

\section{Análise dos dados}

Após a revisão dos dados coletados, eles foram analisados com a utilização do programa SPSS 8.0. As variáveis foram agrupadas por categorias quando necessário, e para os testes de hipótese foram utilizados o teste do Qui-quadrado, o Teste Exato de Fischer e Razão de Chances, com nível de significância de $5 \%(\mathrm{p}<0,05)$ e in- 
tervalo de confiança de 95\%. A razão de chances ajustada foi calculada para algumas variáveis por intermédio de regressão logística. Tais variáveis foram escolhidas baseando-se nas análises bivariadas e no modelo teórico de exposição a situações de risco proposto pelo primeiro autor 9 . Mesmo sendo um estudo transversal, optou-se por utilizar razão de chances para estimativa de risco a fim de que se pudesse testar o modelo teórico proposto.

\section{Resultados}

A prevalência de soropositividade para HIV foi de $22,6 \%$ (95 indivíduos infectados). As características demográficas da amostra, estratificadas quanto à soropositividade HIV, são especificadas na Tabela 2. Observa-se que indivíduos soropositivos e negativos foram semelhantes em relação, ao sexo e ao estado marital. Por outro lado, os soropositivos mais corriqueiramente não trabalhavam e tinham renda inferior a um salário mínimo.

Em relação a comportamentos de risco ( $\mathrm{Ta}-$ bela 3), percebe-se alta chance de soropositividade em relações homossexuais sem uso de preservativo e a menor chance em relação heterossexual desprotegida. O uso de drogas injetáveis foi mais recorrente entre os indivíduos soropositivos, bem como a freqüência a locais específicos para uso de drogas. Note-se uma grande preocupação dos indivíduos soropositivos em já ter sido contaminado e em transmitir o vírus. O uso de drogas no mês anterior e a relação com soropositividade estão descritos na Tabela 4. Somente o uso de cocaína injetável esteve relacionado com infecção por HIV.

Valendo-se dos resultados encontrados nas análises bivariadas, e de acordo com o modelo

Tabela 2

Características demográficas da amostra estudada.

\begin{tabular}{|c|c|c|c|c|c|}
\hline \multirow[t]{2}{*}{ Variável } & \multicolumn{2}{|c|}{ Geral $(n=420)$} & \multicolumn{2}{|c|}{$\mathrm{HIV}+(\mathrm{n}=95)$} & \multirow[t]{2}{*}{$\mathrm{p}$} \\
\hline & $\mathrm{n}$ & $\%$ & $\mathrm{n}$ & $\%$ & \\
\hline \multicolumn{6}{|l|}{ Idade (em anos) } \\
\hline$<20$ & 67 & 16,1 & 10 & 14,9 & \\
\hline $20-29$ & 185 & 44,6 & 40 & 21,6 & 0,24 \\
\hline 30 ou mais & 163 & 39,3 & 44 & 27,0 & $<0,05$ \\
\hline \multicolumn{6}{|l|}{ Gênero } \\
\hline Homem & 292 & 69,5 & 69 & 23,6 & 0,46 \\
\hline Mulher & 128 & 30,5 & 26 & 20,3 & \\
\hline \multicolumn{6}{|l|}{ Estado marital } \\
\hline Solteiro ou sem companheiro/a regular & 195 & 46,7 & 41 & 21,0 & 0,93 \\
\hline Casado ou com companheiro/a regular & 173 & 36,6 & 39 & 25,5 & \\
\hline Outra situação conjugal & 70 & 16,7 & 15 & 21,4 & 0,85 \\
\hline \multicolumn{6}{|l|}{ Situação profissional } \\
\hline Trabalho sistemático & 223 & 53,1 & 41 & 18,5 & \\
\hline Trabalho irregular (não sistemático) & 113 & 27,0 & 27 & 23,9 & 0,24 \\
\hline Não trabalha & 83 & 19,9 & 27 & 32,5 & $<0,05$ \\
\hline \multicolumn{6}{|l|}{ Escolaridade (em anos de estudo) } \\
\hline$<7$ & 171 & 40,8 & 54 & 31,6 & $<0,001$ \\
\hline $8-11$ & 108 & 25,8 & 21 & 19,4 & 0,21 \\
\hline 12 ou mais & 140 & 33,4 & 19 & 13,6 & \\
\hline \multicolumn{6}{|l|}{ Renda (em salários mínimos) } \\
\hline$<1$ & 84 & 20,0 & 30 & 35,7 & $<0,001$ \\
\hline $1-3$ & 161 & 38,3 & 39 & 24,2 & $<0,05$ \\
\hline 4 ou mais & 167 & 39,8 & 24 & 14,4 & \\
\hline
\end{tabular}


Exposição a situações de risco e preocupação quanto à contaminação por HIV e soropositividade.

\begin{tabular}{|c|c|c|c|c|c|c|}
\hline \multirow[t]{2}{*}{ Exposição desde 1980 , alguma vez* } & \multicolumn{2}{|c|}{ Geral $(n=420)$} & \multicolumn{2}{|c|}{$\mathrm{HIV}+(\mathrm{n}=95)$} & \multirow[t]{2}{*}{$p$} & \multirow{2}{*}{$\begin{array}{l}\text { Razão de chances } \\
\text { (IC95\%) }\end{array}$} \\
\hline & $\mathrm{n}$ & $\%$ & $\mathrm{n}$ & $\%$ & & \\
\hline \multicolumn{7}{|l|}{ Transfusão sangüínea } \\
\hline Sim & 25 & 6,0 & 8 & 32,0 & 0,25 & $1,7(0,6-4,2)$ \\
\hline Não & 395 & 94,0 & 87 & 22,0 & & 1 \\
\hline \multicolumn{7}{|l|}{ Relação heterossexual sem preservativo } \\
\hline $\operatorname{Sim}$ & 328 & 78,1 & 52 & 15,8 & $<0,001$ & $0,2(0,1-0,4)$ \\
\hline Não & 92 & 21,9 & 43 & 46,7 & & 1 \\
\hline \multicolumn{7}{|l|}{ Relação homossexual sem preservativo } \\
\hline Sim & 38 & 9,1 & 14 & 36,8 & $<0,05$ & $2,2(1,01-4,6)$ \\
\hline Não & 382 & 90,9 & 81 & 21,2 & & 1 \\
\hline \multicolumn{7}{|l|}{ Relação sexual com profissional de sexo } \\
\hline Sim & 63 & 15,0 & 16 & 25,4 & 0,57 & $1,2(0,6-2,3)$ \\
\hline Não & 357 & 85,0 & 79 & 22,1 & & 1 \\
\hline \multicolumn{7}{|l|}{ Relação sexual por dinheiro } \\
\hline Sim & 37 & 8,8 & 6 & 16,2 & 0,33 & $0,6(0,2-1,7)$ \\
\hline Não & 383 & 91,2 & 89 & 23,2 & & 1 \\
\hline \multicolumn{7}{|l|}{ Uso de droga injetável } \\
\hline Sim & 129 & 30,7 & 57 & 60,0 & $<0,001$ & $5,3(3,2-8,8)$ \\
\hline Não & 291 & 291 & 38 & 13,1 & & 1 \\
\hline \multicolumn{7}{|l|}{ Parceiro/a usuário de droga injetável } \\
\hline Sim & 78 & 18,6 & 21 & 26,9 & 0,31 & $1,3(0,7-2,4)$ \\
\hline Não & 342 & 81,4 & 74 & 21,6 & & 1 \\
\hline \multicolumn{7}{|c|}{$\begin{array}{l}\text { Freqüentar lugar específico para uso de drogas } \\
\text { ao menos } 1 \text { vez (últimos } 6 \text { meses) }\end{array}$} \\
\hline Sim & 186 & 44,3 & 54 & 29,0 & $<0,05$ & $1,9(1,2-3,1)$ \\
\hline Não & 234 & 55,7 & 41 & 21,6 & & 1 \\
\hline \multicolumn{7}{|c|}{ Uso de preservativos nunca/raramente (últimos 6 meses) } \\
\hline Sim & 228 & 54,3 & 56 & 24,6 & 0,30 & $1,3(0,8-2,1)$ \\
\hline Não & 192 & 45,7 & 39 & 20,3 & & 1 \\
\hline \multicolumn{7}{|l|}{$\begin{array}{l}\text { Preocupa-se em já ter sido contaminado } \\
\text { quase sempre/sempre }\end{array}$} \\
\hline Sim & 152 & 36,2 & 52 & 34,2 & $<0,001$ & $2,7(1,7-4,5)$ \\
\hline Não & 268 & 63,8 & 43 & 16,0 & & 1 \\
\hline \multicolumn{7}{|l|}{$\begin{array}{l}\text { Preocupa-se em vir a se contaminar } \\
\text { quase sempre/sempre }\end{array}$} \\
\hline Sim & 185 & 44,1 & 40 & 21,6 & 0,66 & $0,9(0,6-1,5)$ \\
\hline Não & 235 & 55,9 & 55 & 23,4 & & 1 \\
\hline \multicolumn{7}{|c|}{ Preocupa-se em transmitir o vírus quase sempre/sempre } \\
\hline Sim & 256 & 61,0 & 77 & 30,1 & $<0,001$ & $3,5(1,9-6,3)$ \\
\hline Não & 164 & 39,0 & 18 & 10,1 & & 1 \\
\hline
\end{tabular}

* Os sujeitos poderiam preencher mais de uma categoria de resposta. 
Uso de drogas nos 30 dias anteriores à entrevista e soropsitividade.

\begin{tabular}{|c|c|c|c|c|c|c|}
\hline \multirow[t]{2}{*}{ Uso até 30 dias prévios } & \multicolumn{2}{|c|}{ Geral $(n=420)$} & \multicolumn{2}{|c|}{$\mathrm{HIV}+(\mathrm{n}=95)$} & \multirow[t]{2}{*}{$\mathrm{p}$} & \multirow{2}{*}{$\begin{array}{l}\text { Razão de chances } \\
\text { (IC95\%) }\end{array}$} \\
\hline & $n$ & $\%$ & $\mathrm{n}$ & $\%$ & & \\
\hline \multicolumn{7}{|l|}{ Maconha } \\
\hline Sim & 238 & 56,6 & 55 & 23,1 & 0,81 & $1,1(0,7-1,7)$ \\
\hline Não & 182 & 43,3 & 40 & 22,0 & & 1 \\
\hline \multicolumn{7}{|l|}{ Cocaína inalada } \\
\hline Sim & 183 & 43,6 & 41 & 22,4 & 0,92 & $0,98(0,6-1,6)$ \\
\hline Não & 237 & 56,4 & 54 & 22,8 & & 1 \\
\hline \multicolumn{7}{|l|}{ Cocaína injetada } \\
\hline Sim & 74 & 17,6 & 35 & 47,3 & $<0,001$ & $4,3(2,4-7,6)$ \\
\hline Não & 346 & 82,4 & 60 & 17,3 & & 1 \\
\hline \multicolumn{7}{|l|}{ Crack } \\
\hline $\operatorname{Sim}$ & 25 & 6,0 & 6 & 24,0 & 0,87 & $1,1(0,4-3,0)$ \\
\hline Não & 395 & 94,0 & 89 & 22,5 & & 1 \\
\hline \multicolumn{7}{|l|}{ Inalantes } \\
\hline Sim & 50 & 11,9 & 12 & 24,0 & 0,80 & $1,2(0,5-2,3)$ \\
\hline Não & 370 & 88,1 & 83 & 22,4 & & 1 \\
\hline \multicolumn{7}{|l|}{ Álcool } \\
\hline Sim & 178 & 42,4 & 38 & 21,3 & 0,59 & $0,9(0,5-1,4)$ \\
\hline Não & 242 & 57,6 & 57 & 23,6 & & 1 \\
\hline \multicolumn{7}{|l|}{ Tranqüilizantes } \\
\hline Sim & 50 & 11,9 & 12 & 24,0 & 0,80 & $1,1(0,5-2,3)$ \\
\hline Não & 345 & 82,1 & 83 & 24,1 & & 1 \\
\hline
\end{tabular}

teórico proposto por Pechansky et al. 11, foram selecionadas para análise multivariada as seguintes variáveis: idade, sexo, escolaridade, renda familiar, experimentação de droga injetável a partir de 1980, presença em local específico para uso de drogas, uso de preservativos em relações sexuais e preocupação com a possibilidade de vir a se contaminar com HIV. Com relação às duas últimas variáveis, optou-se por incluí-las no modelo multivariado, ainda que não obtendo significância estatística nas análises bivariadas; isso porque ambas têm importância clínico-epidemiológica e são sustentadas pela literatura e pelo modelo etiológico proposto. Calcularam-se razões de chances bruta e ajustada que se encontram expressas na Tabela 5. Percebe-se que foi associado com um maior risco de infecção ter mais de trinta anos, ter renda familiar de menos de um salário mínimo, ter estudado menos de sete anos e ter injetado droga alguma vez desde 1980. Salientase também que o não uso de preservativos apa- rece como fator de proteção para infecção. As análises ajustadas para o sexo do indivíduo e preocupação com contaminação não demonstraram diferença significativa para o desfecho em questão.

\section{Discussão}

Inicialmente, deve-se salientar que este estudo apresenta algumas limitações pela escolha de um modelo epidemiológico não convencional de amostragem. A amostragem pelo sistema de bola de neve, mesmo sendo considerada a mais viável (pois populações escondidas, ou de difícil acesso, como usuários de drogas - em particular UDI - não são acessíveis à epidemiologia convencional, como descrito por Siqueira et al. 12), gera vieses importantes na validade externa de estudos com esta característica. A amostra também é proveniente de centros de saúde, o que pressupõe uma dose significativa de mo- 
Associação entre soropositividade para HIV e as principais variáveis estudadas, utilizando-se como referência para uso de drogas o uso a partir de 1980.

\begin{tabular}{|c|c|c|}
\hline Variável & RC bruta (IC95\%) & RC ajustada (IC95\%) \\
\hline \multicolumn{3}{|l|}{ Gênero } \\
\hline Feminino & 1,00 & 1,00 \\
\hline Masculino & $1,21(0,70-2,11)$ & $0,80(0,43-1,51)$ \\
\hline \multicolumn{3}{|l|}{ Idade (em anos) } \\
\hline$<20$ & 1,00 & 1,00 \\
\hline $20-29$ & $1,57(0,70-3,61)$ & $1,98(0,82-4,76)$ \\
\hline 30 ou mais & $2,11(0,94-4,85)$ & $2,89(1,17-7,12)$ \\
\hline \multicolumn{3}{|l|}{ Anos de estudo } \\
\hline Até 7 & $2,94(1,59-5,49)$ & $2,10(1,02-4,36)$ \\
\hline $8-11$ & $1,54(0,74-3,20)$ & $1,04(0,46-2,35)$ \\
\hline 12 ou mais & 1,00 & 1,00 \\
\hline \multicolumn{3}{|l|}{ Renda (em salários mínimos) } \\
\hline$<1$ & $3,31(1,70-6,46)$ & $2,89(1,32-6,32)$ \\
\hline $1-3$ & $1,90(1,05-3,48)$ & $1,51(0,76-2,98)$ \\
\hline 4 ou mais & 1,00 & 1,00 \\
\hline \multicolumn{3}{|l|}{ UDI a partir de 1980} \\
\hline Não & 1,00 & 1,00 \\
\hline $\operatorname{sim}$ & $5,27(3,15-8,84)$ & $5,18(2,89-9,28)$ \\
\hline \multicolumn{3}{|c|}{ Freqüencia a locais para uso de drogas } \\
\hline Nunca & 1,00 & 1,00 \\
\hline Raramente & $1,79(0,99-3,24)$ & $1,89(0,96-3,70)$ \\
\hline Várias vezes por mês & $2,47(1,26-4,84)$ & $1,78(0,85-3,73)$ \\
\hline Todos ou quase todos os dias & $1,34(0,45-3,81)$ & $0,64(0,21-1,91)$ \\
\hline \multicolumn{3}{|l|}{ Uso de preservativos } \\
\hline Quase sempre/sempre & 1,00 & 1,00 \\
\hline Nunca/raramente & $1,23(0,75-200)$ & $0,57(0,33-0,99)$ \\
\hline \multicolumn{3}{|c|}{ Preocupação com contaminar-se no futuro } \\
\hline Quase sempre/sempre & 1,00 & 1,00 \\
\hline Nunca/raramente & $0,97(0,59-1,59)$ & $1,25(0,72-2,16)$ \\
\hline
\end{tabular}

UDI = usuários de drogas injetáveis.

tivação para comparecer à consulta, e os indivíduos referidos pelos sujeitos-chave podem ser indivíduos mais graves do ponto de vista de abuso de drogas ou risco para HIV.

Em quase $40,0 \%$ dos indivíduos não obtivemos o resultado do exame anti-HIV e isso se deveu sobretudo a problemas de logística nos locais de coleta. Essa perda pode ter gerado um viés de seleção se considerarmos que existiam algumas diferenças entre esses indivíduos, especialmente com relação ao sexo e uso de droga injetável. O fato de os indivíduos excluídos serem provenientes, em sua maioria, de dois centros de tratamento para dependência química pode ter ocasionado a exclusão dos indivíduos com uso mais problemático de drogas. Por outro lado, os indivíduos que buscavam testagem para HIV nos COAS possivelmente fossem usuários de drogas menos graves, já que não necessariamente estavam em tratamento, o que pode ter gerado a falta de associação entre o uso de drogas e maior chance de contaminação por HIV, que só apareceu no uso de droga injetável.

Quanto ao instrumento, o CRA estava em adaptação para o meio brasileiro e teve uma 
seção inteira retirada de sua forma original, atitude que modifica a amplitude dos escores possíveis e impede sua comparação direta com estudos norte-americanos, o que está descrito em outro artigo 12. Uma das questões retiradas do questionário foi a que se refere ao uso compartilhado de seringas, pois não se tinha estimativa apropriada sobre a real prevalência de UDI na população estudada, e o estudo piloto realizado para a validação do instrumento não demonstrou a magnitude do problema. Ainda assim, os autores consideram que a originalidade dos achados supera as limitações do estudo em questão, devido à escassez de dados de pesquisa existentes em nosso meio.

Pode-se perceber que uma parcela significativa dos entrevistados apresentava renda familiar acima de três salários mínimos, contradizendo a impressão empírica de que usuários de drogas se concentram em uma faixa populacional de renda muito baixa ou inexistente. Outro ponto importante é que trata-se de uma população jovem e potencialmente produtiva, como também demonstram Lima et al. 8, Barcellos 13 e Castilho et al. 14 entre outros, que identificam indivíduos dos 25 aos 40 anos como a faixa de maior concentração de usuários de drogas sob risco de soropositividade para o HIV.

Quanto à soropositividade e testagem HIV, importa ressaltar que a taxa encontrada é bastante alta $(22,6 \%)$, especialmente porque os indivíduos estudados eram usuários de drogas variadas e não apenas drogas injetáveis e porque 70,0\% nunca haviam injetado drogas. Entretanto, é provável que a forma de uso e não o tipo de droga esteja associada com maior risco, uma vez que apenas o uso injetável de cocaína apresentou diferença significativa quanto à soropositividade do respondente. A relação entre uso de drogas injetáveis e soropositividade já é bem estabelecida na literatura por Des Jarlais et al. 15 e Nwanyanwu et al. 16.

Os indivíduos com menor escolaridade estavam mais infectados por HIV do que os com mais anos de estudo. Logo, parece que genericamente estudar mais - e assim estar mais apto a receber e manusear informações - estaria associado com menor soropositividade. Com relação à idade, os dados demonstram que os indivíduos mais velhos têm maior chance de serem soropositivos, o que se explicaria por uma maior exposição a comportamentos repetidos de risco ao longo da vida, como sexo desprotegido e o uso de drogas injetáveis. Ambos os achados são compatíveis com o modelo de risco utilizado para análise dos dados Pechansky ${ }^{9}$.

O estudo não mostrou diferença significativa entre homens e mulheres na chance de in- fectar-se por HIV. Isto pode estar refletindo a realidade do país e do mundo, dado que a proporção de mulheres infectadas vem aumentando muito nos últimos anos, de acordo com diversos relatos epidemiológicos, inclusive brasileiros, como o Boletim Epidemiológico AIDS (abril a setembro de 2002 - http: / /www.aids. gov.br, acessado em 07/Jul/2003). Cabe observar também que, devido às mulheres utilizarem mais os centros de saúde em geral, como mostram Llorente-Alvarez et al. 17 e ManceraRomero et al. 18, é provável que a população feminina desses centros tenha menor risco de contaminação que os homens, considerando que fariam testagem mais precocemente em termos de riscos. Todavia, como em nosso estudo só foram incluídos indivíduos com algum uso de drogas, é provável que tais diferenças tenham diminuído, levando em conta que a amostra final constitui-se na maioria por homens, permanecendo provavelmente apenas as mulheres de maior risco, fato que também nos ajudaria a entender a taxa de contaminação por HIV similar à do sexo masculino.

A comparação entre os valores brutos e os ajustados na análise multivariada basicamente confirma o modelo proposto: há uma associação direta entre idade e risco - significativa para a faixa mais velha - e inversa entre as variáveis escolaridade e renda versus o risco de soropositividade. A freqüência (qualquer) a locais para consumo de drogas apresentou uma razão de chance para soropositividade significativamente maior do que a opção "nunca”. Contudo, este é um achado que precisa ser melhor explorado em análises posteriores, provavelmente com a inclusão de outros elementos informativos, já que a análise dos valores ajustados mostra uma perda da significância estatística. O não uso de preservativos surgir como fator de proteção pode estar associado ao fato de tratar-se de um estudo transversal e muitos indivíduos já saberem-se previamente soropositivos, preocupando-se mais em usar preservativos. Friedman et al. 1 em um estudo transversal com UDI demonstram que o uso de preservativos estava associado com soropositividade.

A variável que mais discrimina o risco de soropositividade, seja de forma isolada, ou após ajuste com outras variáveis, é o uso de drogas injetáveis. Este não é um achado novo: Guimarães \& Castilho 19, Inciardi et al. 20 e Lima et al. 8, dentre outros autores que realizaram estudos no Brasil, já haviam confirmado esta forte associação, que é conhecida em praticamente todos os estudos realizados com este tipo de população no mundo. A importância do achado se refere às características locais e à 
escassez de informações empíricas em nosso meio.

Mesmo com os viéses de seleção já descritos nos comentários anteriores, este estudo teve como finalidade ilustrar uma realidade clinicamente conhecida, mas cujos números ainda não eram identificados. A alta taxa de infecção encontrada, tanto em UDI quanto nos que utilizam droga por outras vias, mostra uma rea- lidade que sugere medidas preventivas eficazes. As análises desse estudo sugerem que diferentes variáveis estão associadas à contaminação pelo HIV, como baixas escolaridade e renda, maior idade e uso de droga injetável, implicando que tais medidas de prevenção sejam mais focadas, levando em consideração as peculiaridades de subgrupos dentre os usuários de drogas.

\section{Resumo}

Em um estudo transversal com uma amostra de 420 usuários de drogas de Porto Alegre, Rio Grande do Sul, Brasil, foram avaliados demografia, uso de drogas e situações de risco para contaminação pelo HIV por meio da versão brasileira do questionário Comportamentos de Risco para AIDS. A prevalência de HIV positivos foi de 22,6\%; 39,3\% tinham 30 anos ou mais e 69,5\% eram homens. Nos trinta dias prévios à coleta, $56,8 \%$ tinham usado maconha, 43,6\% cocaína inalada, 17,6\% cocaína injetada e 42,4\% álcool freqüentemente. As variáveis que se mantiveram associadas com infecção por HIV após regressão logística foram idade superior a trinta anos (RC: 2,89; IC95\%: 1,17-7,12), ter menos de sete anos de estudo (RC: 2,10; IC95\%: 1,024,36), renda de menos de um salário mínimo (RC: 2,89; IC95\%: 1,32-6,32) e ter usado droga injetável (DI) (RC: 5,18; IC95\%: 2,89-9,28). A taxa de infecção pelo HIV encontrada foi alta, considerando que 70,0\% nunca haviam usado DI. As variáveis associadas com contaminação por HIV estão de acordo com as literaturas nacional e internacional e com o modelo teórico de exposição a risco proposto pelo primeiro autor.

HIV; Síndrome de Imunodeficiência Adquirida; Drogas Ilícitas; Fatores de Risco

\section{Colaboradores}

F. Pechansky coordenou a execução do projeto de pesquisa e a confecção das diversas etapas deste artigo. L. von Diemen realizou a análise dos dados e a preparação das tabelas, bem como a confecção da discussão do artigo. H. Surratt e J. Inciardi coordenaram o projeto de pesquisa que originou os dados deste estudo, fazendo sugestões e adaptando o texto final ao modelo de publicação proposto. R. De Boni organizou a revisão bibliográfica e participou da elaboração da discussão e introdução do artigo.

\section{Agradecimentos}

O financiamento para o projeto que serviu de base para que este artigo fosse escrito foi obtido por meio do projeto de pesquisa Assessment of Behaviors for HIV Transmission among High Risk Individuals in Porto Alegre, da Universidade de Delaware, Estados Unidos. 


\section{Referências}

1. Friedman SR, Benny J, Neaigus A, Golstein M, Curtis R, Ildefonso G, et al. Consistent condom use in relationships between seropositive injecting drug users and sex partners who do not inject drugs. AIDS 1994; 8:357-61.

2. Anderson JE, Wilson RW, Barker P, Doll L, Jones TS, Holtgrave D. Prevalence of sexual and drugrelated HIV risk behaviors in the U.S. adult population: results of the 1996 National Household Survey on Drug Abuse. J Acquir Immune Defic Syndr 1999; 21:148-56.

3. Edlin BR, Irwin KL, Faruque S, McCoy CB, Word $\mathrm{C}$, Serrano Y, et al. Intersecting epidemics-crack cocaine use and HIV infection among inner-city young adults. N Engl J Med 1994; 331:1422-7.

4. Inciardi JA, Lockwood D, Pottieger AE. Women and crack-cocaine. New York: McMillan Publishing Co.; 1993.

5. Pechansky F, von Diemen L. Homens e mulheres usuários de drogas: semelhanças e diferenças na exposição ao vírus HIV em Porto Alegre. Revista da ABEAD 1998; 1/2:15-25.

6. Pechansky F, Inciardi JA, Surrat H, Lima AF, Kessler FHP, Soilbelman M, et al. Estudo sobre as características de usuários de drogas injetáveis que buscam atendimento em Porto Alegre, RS. Rev Bras Psiquiatr 2000; 22:164-71.

7. Pechansky F, von Diemen L, Genro V. Presença de situações de risco para a transmissão do HIV em usuários de drogas não injetáveis. Rev Psiquiatr Clín (São Paulo) 2001; 28:120-5.

8. Lima ES, Friedman SR, Bastos FI, Telles PR, Friedmann P, Ward TP, et al. Risk factors for HIV-1 seroprevalence among drug injectors in the cocaine using environment of Rio de Janeiro. Addiction 1994; 89:689-98.

9. Pechansky F. Modelo teórico de exposição a risco para transmissão do vírus HIV em usuários de drogas. Rev Bras Psiquiatr 2001; 23:41-7.

10. Metzger D, Dephilippis D, Druley P. The impact of HIV testing on risk for AIDS behaviors. In: Harris LS, editor. Problems of drug dependence. Washington DC: National Institute on Drug Abuse; 1992. p. 297-8. (NIDA Research Monograph 119).
11. Pechansky F, Metzger D, Hirakata V. Adaptação e validação de um questionário sobre comportamento de risco para AIDS em usuários de drogas em Porto Alegre. Rev Bras Psiquiatr 2002; 24:130-6.

12. Siqueira D. O projeto de redução de danos no Rio Grande do Sul. In: Bastos FI, Mesquita F, Marques LF, organizadores. Troca de seringas: drogas e AIDS. Brasília: Ministério da Saúde; 1998.

13. Barcellos N. AIDS no Estado do Rio Grande do Sul: avaliação epidemiológica dos casos notificados até 31 de março de 1998. Porto Alegre: Seção de Controle de AIDS, Departamento de Ações em Saúde, Secretaria da Saúde e do Meio Ambiente; 1998.

14. Castilho EA, Szwarcwald CL. Situação atual da AIDS no Brasil. Diagn Tratamento 1997; 2:17-20.

15. Des Jarlais DC, Friedman SR, Stoneburner RL. HIV infection and intravenous drug use: critical issues in transmission dynamics, infection outcomes, and prevention. Rev Infect Dis 1988; 10: 151-8.

16. Nwanyanwu OC, Chu SY, Green TA, Buehler JW, Berkelman RL. Acquired immunodeficiency syndrome in the United States associated with injecting drug use, 1981-1991. Am J Drug Alcohol Abuse 1993; 19:399-408.

17. Llorente-Álvarez S, López-Ruiz T, García-Lavandera LJ, Alonso-Arias P, Muñoz-Baragaño P, Alonso-Fernández M. Perfil del hiperfrecuentador de un centro de salud. Aten Primaria 1996; 17:100-6.

18. Mancera-Romero J, Muñoz-Cobos F, PaniaguaGómez F, Fernández-Lozano C, Fernández-Tapia M, Blanca-Barba F. Problemas de salud y factores determinantes del número de visitas a demanda en pacientes hiperutilizadores de un centro de salud. Aten Primaria 2001; 27:658-62.

19. Guimarães MD, Castilho EA. Aspectos epidemiológicos da AIDS/HIV no Brasil. Rev Soc Bras Med Trop 1993; 26:101-11.

20. Inciardi JA, Surratt HL, McCoy HV. Establishing and HIV/AIDS intervention program for street drug users in a developing nation. J Drug Issues 1997; 27:173-93.

Recebido em 27/Set/2002

Versão final reapresentada em 05/Abr/2004

Aprovado em 03/Ago/2004 